\section{Lessons from viral manipulation of protein disposal pathways}

\author{
Margo H. Furman and Hidde L. Ploegh \\ Department of Pathology, Harvard Medical School, Boston, \\ Massachusetts, USA \\ J. Clin. Invest. 110:875-879 (2002). doi:10.1172/JCI200216831.
}

Detection and elimination of viral pathogens is a continual challenge for all organisms, even mammals which benefit from a sophisticated immune system. Many classes of viruses are resistant to complete elimination by the immune system and persist in a latent or minimally replicative state for the lifetime of the host. This persistence is often without clinical consequence to the host, as extensive damage would compromise survival of the pathogen. Examination of viral gene products has revealed a multitude of strategies employed by viruses to escape from innate and adaptive host defense mechanisms. These features enable long-term infection and create a delicate balance between the virus's ability to replicate and spread and the host's ability to control these events.

Although viruses replicate inside host cells, their presence is not concealed from the watchful eye of the immune system. Numerous cellular mechanisms provide protection where mechanical and humoral defenses cannot. NK cells are among the first lymphocytes to sense the release of IFN- $\alpha$ and IFN- $\beta$, as well as perturbations in expression of MHC class I molecules and other surface molecules, all of which are triggered by viral invasion of cells. Several days after exposure to the virus, specific CTLs become activated. These CTLs, recognizing viral antigenic peptides bound to host cell MHC class I molecules, then begin to eliminate infected cells by releasing cytolytic and proapoptotic factors like perforin and granzyme, IFN- $\gamma$, TNF- $\alpha$, and TNF- $\beta$ (1). This system is essential to root out intracellular pathogens, as demonstrated by the increased susceptibility of animals lacking CTLs to many types of pathogens, particularly viruses (2).

Address correspondence to: Hidde L. Ploegh, Harvard Medical School, 200 Longwood Avenue, Armenise Building, Room 137, Boston, Massachusetts 02115, USA. Phone: (617) 432-4777; Fax: (617) 432-4775; E-mail: ploegh@hms.harvard.edu.

Conflict of interest: No conflict of interest has been declared. Nonstandard abbreviations used: endoplasmic reticulum (ER); human cytomegalovirus (HCMV); human T cell leukemia virus type-1 (HTLV-1); plant homeodomain (PHD); murine $\gamma$-herpesvirus-68 (MHV-68); human herpesvirus-8 (HHV-8); myxoma virus leukemia-associated protein (MV-LAP); enhanced green fluorescent protein (EGFP).
The MHC class I pathway provides numerous points of interference for viral pathogens. This is logical, as MHC class I proteins continually sample the intracellular space inhabited by viruses and present this intracellular material on the surface of host cells for recognition by CTLs. The remarkable variety of proteins encoded by viruses to avoid CTL recognition shows that this arm of the adaptive immune system must be disabled or circumvented by successful intracellular pathogens. At the same time, the sheer diversity of molecular mechanisms by which CTL avoidance is achieved makes it difficult to predict how newly identified immune-evasive molecules accomplish this end (see refs. 3, 4 for recent reviews). Here, we discuss new data and unresolved questions in this exciting field.

\section{MHC class I biosynthesis and antigen presentation} Figure 1 depicts key steps in the synthesis and assembly of MHC class I complexes. An MHC class I complex, as it exists on the cell surface, consists of a $46-\mathrm{kDa}$ transmembrane glycoprotein subunit (also called the heavy chain), noncovalently associated with $\beta_{2}$ microglobulin $\left(\beta_{2} \mathrm{~m}\right)$ and tightly complexed with a peptide of eight to ten amino acids. The heavy chain and $\beta_{2}$ m subunits are inserted cotranslationally into the endoplasmic reticulum (ER), where they also acquire peptide ligands. These peptides are generated primarily in the cytosol by the proteasomal cleavage of cellular or viral proteins.

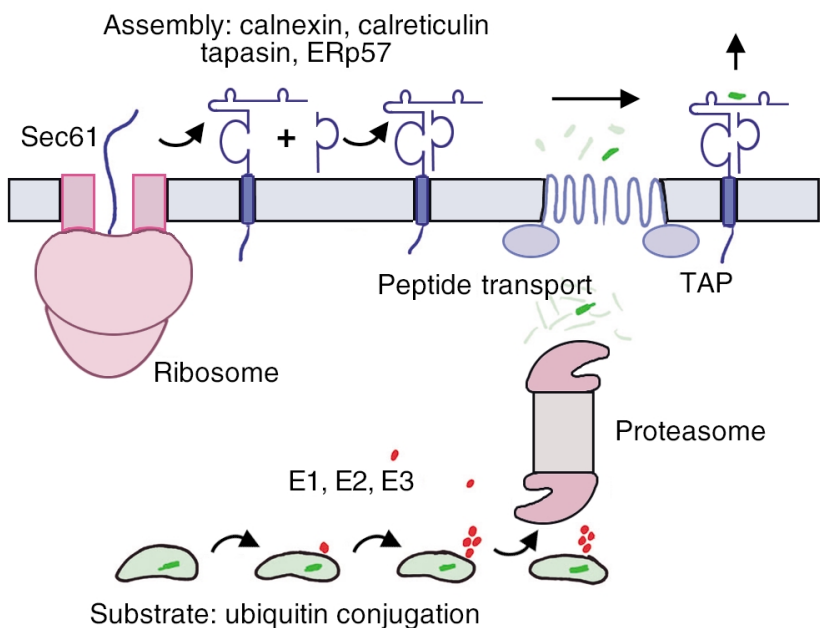

Figure 1

MHC class I complex synthesis and assembly. MHC class I heavy chains are cotranslationally inserted into the ER. Proper folding, association with the $\beta_{2} \mathrm{~m}$ light chain, and peptide loading are assisted by chaperones such as calnexin, calreticulin, tapasin, and ERp57. E1, E2, and E3 ubiquitin-conjugating enzymes tag proteins in the cytosol for destruction by the proteasome. Peptides are pumped into the ER by the TAP transporters. Properly folded, peptide-loaded MHC class I complexes progress through the secretory pathway for display on the cell surface. TAP, transporter associated with antigen processing. 
Appropriate folding of nascent heavy chains is assisted by several chaperones. Association of heavy chain with $\beta_{2} \mathrm{~m}$ results in release of the transmembrane chaperone calnexin and association with another chaperone, the soluble calnexin homologue calreticulin. After association with calreticulin, heavy chain $/ \beta_{2}$ m dimers are ready to acquire peptide, which is transported into the ER by the TAP1 and TAP2 transmembrane ATPases (transporters associated with antigen processing). A third chaperone, tapasin, mediates the association between heavy chain $/ \beta_{2}$ m dimers and TAP, allowing the efficient loading of transported peptides. Yet another chaperone, the thiol-dependent oxidoreductase ERp57, participates in MHC class I complex formation, perhaps by cooperation with tapasin (5). Acquisition of suitable peptide cargo confers stability on the complex, which is then transported to the cell surface by the secretory pathway (6). $\mathrm{CD} 8^{+} \mathrm{T}$ cells are specialized to recognize these surface complexes when they present peptides derived from microbial sources. Recognition results in specific lysis of the infected target cell by the CTL and/or the directed release of cytotoxic cytokines. Each step in the assembly of class I molecules is subject to quality control; the absence or even a reduction in levels of any of the accessory subunits reduces surface expression of the other components (7).

\section{Viral avoidance of MHC class I antigen presentation}

The human cytomegalovirus (HCMV) US2 and US11 gene products bind to nascent class I heavy chains and remove them from the $\operatorname{ER}(8,9)$. Once in the cytosol, they are promptly degraded by the proteasome, as discussed below. In addition, the HCMV US3 protein targets ER-resident heavy chains and simply prevents their egress from the ER in a mechanism that relies on cycles of binding and release (10-12). Herpes simplex viruses- 1 and -2 encode a protein, ICP47, that blocks the TAP transporters from the cytosolic side of the ER $(13,14)$. HCMV encodes yet an additional protein, US6, which also targets TAP1 and TAP2, but from the lumenal side of the ER $(15,16)$. One of the main Epstein-Barr virus proteins expressed during latency, EBNA1, is itself resistant to proteolysis by the proteasome, and thus peptides derived from EBNA1 are not generated and detected by CTLs (17).

Even viruses with more compact genomes encode proteins with similar functions. One notable example is the Nef protein encoded by HIV, which promotes the endocytosis of CD4, the coreceptor for $\operatorname{HIV}(18,19)$. Nef also downmodulates surface expression of the MHC class I alleles human leukocyte antigen-A (HLA-A) and human leukocyte antigen-B (HLA-B), apparently by acting in the Golgi apparatus to target these class I molecules to lysosomes $(20,21)$. A recent report shows that Nef may also disrupt expression of mature MHC class II molecules by an unknown mechanism, perhaps indirectly (22).

By virtue of their rapid replication rates and small genome sizes, retroviruses rely heavily on mutational escape to avoid immune detection. Unlike herpesviruses, which establish latency, retroviruses replicate continually in the host. In addition to the HIV
Nef-dependent downmodulation of MHC class I and perhaps class II molecules, a new MHC class I downregulatory molecule has been reported for another human retrovirus. The $\mathrm{p} 12$ protein, found in the ER and Golgi apparatus of human $\mathrm{T}$ cell leukemia virus type-1-infected (HTLV-1-infected) cells, binds nascent MHC class I molecules and causes their proteasomal destruction. Interestingly, the $\mathrm{p} 12$ protein has two natural variants that correlate with differences in pathogenesis. The $\mathrm{p} 12$ with a lysine at position 88 is found in patients with an immunological disorder called tropical spastic paraparesis/HTLV-1-associated myelopathy (TSP-HAM). The version with an arginine at position 88 , however, is found in healthy carriers and adult $T$ cell leukemia patients $(23,24)$. The lysine version is unstable, likely due to ubiquitination (24). It will be important to identify the host factors that interact with p12 and stimulate its own turnover and turnover of MHC class I molecules, and to investigate the role of the variants in contributing to different human pathologies.

Many of these immuno-evasive proteins are dispensable for replication in vitro but most likely contribute to virulence in vivo. Because their effects do not require additional virally encoded proteins, it is generally assumed that normal cellular pathways are used to execute the immunoevasin's function. The diversity of viral immunoevasins and their targets must reflect similar heterogeneity in the routes by which cells dispose of proteins that are no longer needed.

\section{Herpesviruses and the ubiquitin system}

The ubiquitin system, which regulates numerous essential cellular processes, presents a wide range of possibilities for viral manipulation. Recent advances in the characterization of ubiquitin-modifying enzymes have converged with the identification of immunoevasins in several $\gamma$-herpesviruses. Many proteins containing zincbinding domains called RING fingers trigger the cytosolic or lysosomal degradation of MHC products. Many RING finger proteins act as E3 ubiquitin ligases (25), suggesting a likely basis for the biological activity of these viral proteins. A related zinc-finger structure, termed a plant homeodomain (PHD) or LAP domain, is found in the murine $\gamma$-herpesvirus-68 (MHV-68) MK3 protein and the human herpesvirus-8 (HHV-8) proteins $\mathrm{K} 3$ and K5 (also known as MIR1 and MIR2, for modulator of immune recognition). All three proteins cause the destruction of MHC class I molecules, with K5 downregulating B7.2 and ICAM-1 as well (26-28).

$\mathrm{K} 3, \mathrm{~K} 5$, and MK3 have several key similarities and differences. In the presence of $\mathrm{K} 3$ or $\mathrm{K} 5$, MHC class I assembly and transport to the cell surface are unaffected. $\mathrm{K} 3$ and $\mathrm{K} 5$ recognize class I via interactions with the transmembrane domains and cause rapid internalization and destruction of class I molecules in an endolysosomal compartment (Figure 2) $(26,28,29)$. The subcellular localization of these viral gene products and the compartment in which they bind class I has been difficult to determine. While the K3/K5-mediated destruction of class I molecules is carried out by lysosomal proteases, ubiquitination of lysine residues evidently plays 


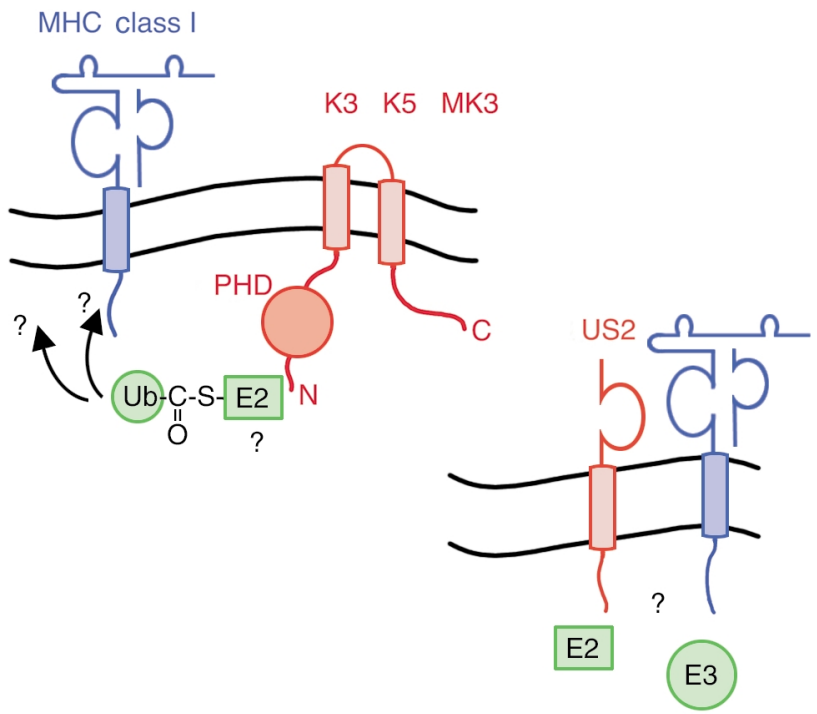

Figure 2

Viral ubiquitination and the destruction of MHC class I molecules. HHV-8 $\mathrm{K} 3$ and $\mathrm{K} 5$, and $\mathrm{MHV}-68 \mathrm{MK} 3$, encode proteins with a RING finger-like $\mathrm{PHD}$ domain that recognizes $\mathrm{MHC}$ class I molecules via interactions between the transmembrane regions of the viral and the host molecule. The $\mathrm{N}$-terminal PHD domain may recruit an E2 enzyme to stimulate ubiquitination, leading to proteasomal destruction in the case of MK3, or endocytosis and lysosomal destruction in the case of $\mathrm{K} 3$ and $\mathrm{K} 5$, respectively. HCMV US2 encodes an Ig-like transmembrane protein that binds to the MHC class I heavy chain in the ER. The short cytosolic tail of US2 is required to stimulate dislocation of heavy chains, perhaps by recruiting ubiquitinating enzymes. Ub, ubiquitin.

a role in targeting the molecules for degradation. Thus, the addition of lysines to the cytosolic tail of B7.1 renders the molecule susceptible to ubiquitination by both $\mathrm{K} 3$ and K5 and to downregulation by K5 (30), whereas removal of the lysines from the cytosolic tail of HLA-B7 prevents its downregulation by K5 (30). Furthermore, the PHD domain of $\mathrm{K} 5$ can mediate ubiquitination in vitro (30). Proteasome inhibition stabilizes class I molecules and blocks their redistribution into dense endocytic compartments in K3-BJAB transfectants, indicating a crucial role for proteasome activity in $\mathrm{K} 3$-triggered sorting (31). It is still unclear how or whether direct ubiquitination of class I molecules leads to their destruction in the presence of $\mathrm{K} 3$ and $\mathrm{K} 5$, although ubiquitination is clearly enhanced by the expression of $\mathrm{K} 3$ and $\mathrm{K} 5$, with class I molecules being possible substrates (30). An intriguing link is suggested by the increase in activity of the proteasome-associated ubiquitin isopeptidase USP14 when the proteasome's catalytic sites are blocked (32). A blockade of proteasomal activity abrogates ubiquitin-dependent internalization of the growth hormone receptor (33). It is therefore possible that the increase in USP14 activity could reverse ubiquitin modifications essential for downregulation.

MK3 of MHV-68 is found in the ER and degrades MHC class I molecules from this compartment in a proteasome-dependent manner (34). Ablation of cytosolic lysines of MHC class I prevents degradation but, curiously, not the appearance of mono- or oligoubiquitinated polypeptides (34). These polypeptides lack the usual long polyubiquitin chains found on cellular proteins that have been targeted for degradation. It will be critical to determine the site of attachment of ubiquitin on these MK3-targeted MHC class I molecules and the intracellular compartment in which this modification occurs.

The role of this ubiquitin modification in $\mathrm{K} 3 / \mathrm{K} 5$ - and MK3-mediated degradation remains uncertain. In yeast and in mammalian cells, ubiquitination contributes to protein sorting to the vacuole and to the endocytosis of certain surface receptors (ref. 35 and references therein). RING finger proteins such as c-Cbl are reported to stimulate endocytosis by ubiquitinating the cytoplasmic domain of some cell surface receptors (36). Because short ubiquitin chains are associated with endocytosis and other nonproteasomal fates (37), modification by K3 and K5 may likewise alter the trafficking of class I molecules between ER, plasma membrane, endosomal, and lysosomal compartments. Thus, while K3, K5, and MK3 all employ PHD domains and are specific for class I molecules, they use distinct pathways to destroy their substrates. Identification of the E2 ubiquitin-conjugating enzymes that enable substrate ubiquitination by these proteins and the localization of the viral gene products may help define the intracellular compartments and proteinases involved in their degradation.

Yet another PHD/LAP protein that downregulates MHC class I molecules has been identified in myxoma virus, a member of the Poxviridae family. Like the K3, $\mathrm{K} 5$, and MK3 proteins, myxoma virus leukemia-associated protein (MV-LAP) appears to be retained in the ER and stimulates rapid endocytosis of class I molecules (38). Truncated MV-LAP that is not retained in the ER fails to downregulate class I molecules. Myxoma virus lacking MV-LAP has reduced virulence. It will be interesting to compare MV-LAP to K3, K5, MK3, and the other PHD-containing proteins in herpes and poxviruses to understand their remarkable ability, as ER-resident proteins, to trigger the internalization of class I molecules and other surface-disposed membrane proteins.

The US2 and US11 gene products of HCMV interact with the ubiquitin system in equally intriguing ways, provoking the dissociation of MHC class I molecules from the ER to the cytosol, where they are destroyed by the proteasome (Figure 2) $(8,9)$. As this sequence of events resembles the removal of misfolded proteins, it provides a model system for understanding the disposal of defective ER proteins more generally (39). Misfolded cellular proteins are disposed of only after a period of retention $(40,41)$, but the elements that recognize them are not clear. On the other hand, US2 and US11 represent defined recognition elements for MHC class I degradation, but the subsequent disposal of these proteins occurs rapidly $(8,9)$. It will be important to clarify the role of ubiquitination in the dislocation of proteins that fail to pass ER quality control standards or that are the substrate of viral attack.

Work in cultured cells suggests that ubiquitination is also required in US11-mediated degradation. Thus, MHC class I molecules expressed in hamster cells with a temperature-sensitive mutation in the E1 ubiquitinactivating enzyme are resistant to US11-mediated 
degradation (42). Moreover, ubiquitin-depleted cytosol fails to support US11-mediated degradation of class I heavy chains in a permeabilized cell system (43), in which polyubiquitinated heavy chains can be detected (44). Heavy chains remain in the ER in the absence of a functional ubiquitin system $(42,43)$, but ablation of cytosolic lysines of MHC class I does not prevent degradation of class I molecules. It remains unclear how the lumenal portion of class I molecules becomes accessible to ubiquitin-conjugating enzymes, but a small fraction of heavy chains is found in association with p97 (also known as valosin-containing protein or $\mathrm{VCP}$ ), a cellular protein that may assist in extracting polyubiquitinated heavy chains from the ER in the presence of US11 (45). Whether direct ubiquitination of heavy chains must occur to cause degradation remains a mystery, as do the relevant ubiquitin-conjugating enzymes. One might in principle be able to show a stoichiometric relationship of ubiquitin conjugation and degradation of class I molecules, which would suggest that each class I molecule to be destroyed requires ubiquitin conjugation; but it is also possible that ubiquitin addition plays a catalytic role, targeting for degradation a number of substrates, perhaps those confined to some membrane microdomain.

Substrate recognition and dislocation are also proposed to depend on cytoplasmic interactions between elements in US2 or US11 and those on the MHC class I substrate. Both US2 and US11 carry short cytosolic tails. While removal of the tail of US2 renders it unable to degrade class I molecules, a similar mutation of US11 has no such effect (46). US2 and US11 are both sensitive to deletion of the cytosolic tail of class I (47). Further modifications of the class I tail indicate lengthbut not sequence-dependent requirements of the class I tail, which differ between US2 and US11 (M. Kim et al., unpublished data). These data suggest a mechanistic difference between US2 and US11 function, the nature of which has not yet been explained, and they raise the important question of apparent redundancy of viral immunoevasins. The polymorphism of the target class I molecules has been invoked as an explanation, but it is also possible that downstream effectors could be expressed in a tissue-specific fashion, thus necessitating mechanistically distinct immunoevasins for different host cell types.

The multiplicity of HCMV-encoded immunoevasins that seemingly carry out the same reaction, culminating in the destruction of MHC class I products, raises the possibility of multiple pathways to achieve the same goal. We consider it likely that mechanisms for disposal of unwanted cellular proteins are also equally diverse.

\section{Structural surprises from viral evasins}

Surprises have emerged from structural studies of some immune-inhibitory viral proteins (48). The structure of the lumenal domain of HCMV US2 protein, complexed with the HLA-A2 allele of MHC class I and $\beta_{2} \mathrm{~m}$, provides several insights into US2 function. The US2 protein itself adopts an Ig-like fold, despite less than 12\% sequence identity with other molecules containing a similar three-dimensional structure. US2 binds to the heavy chain at the junction between the peptide-binding cleft and the $\alpha 3$ domain (49). Interestingly, all membrane protein ligands of MHC class I for which the structure has been solved - including the $\alpha \beta T$ cell receptor, an Ig-like NK cell receptor; CD8 $\alpha \alpha$, a lectinlike NK receptor; and HCMV US2 - bind to nonoverlapping sites on MHC class I. US2 binds stably to the MHC class I complex without affecting the latter's conformation (49). Although it clearly does not destabilize the complex by causing it to unfold, it may recruit other components to initiate dislocation $(48,49)$. Identification of those players remains a major challenge.

The stability of the US2-class I complex also raises questions as to the sequence of events in the dislocation reaction. US2 assembles with fully folded class I molecules in the ER, the very site from which dislocation is initiated. Is disassembly of the complex required? If so, which are the catalysts involved? Could the entire complex be discharged through a proteinaceous channel, only to be disassembled upon arrival in the cytosol? We have made enhanced green fluorescent protein-class I (EGFP-class I) fusion proteins that continue to be dislocated in a US2- and US11-dependent fashion. Upon inhibition of proteasomal proteolysis, we observe accumulation of fluorescent fusion protein in the cytosol (50). While transient unfolding of the EGFP moiety cannot be excluded, these data would be consistent with dislocation of the folded EGFP entity. Again, these observations raise important questions about the types and folding states of substrates that can be dislocated.

\section{Conclusions}

There is no shortage of viral proteins to illustrate the complexity of host/pathogen interactions and the diversity with which these interactions can be modulated. The PHD/LAP proteins encoded by herpes- and poxviruses have much to teach us about the subtleties of ubiquitin-mediated sorting and regulation. Murine and rabbit models highlight the importance of immune evasins in vivo, as shown by infections with viruses lacking these proteins. A key finding is the multiplicity of viral functions that target MHC glycoproteins. The structural diversity of the proteins that constitute this set of immunoevasins suggests a corresponding complexity for the cellular pathways targeted. The elucidation of viral structures will continue to provide important details about the mechanisms by which host immunity is compromised and may clarify the relationships with cellular pathways of quality control and protein turnover in mammalian cells.

\section{Acknowledgments}

This work was funded by NIH grants 5R37-AI33456 and P01-AI42257. M.H. Furman is an Albert J. Ryan Fellow.

1. Shresta, S., Pham, C.T., Thomas, D.A., Graubert, T.A., and Ley, T.J. 1998 How do cytotoxic lymphocytes kill their targets? Curr. Opin. Immunol. 10:581-587.

2. Harty, J.T., Tvinnereim, A.R., and White, D.W. 2000. CD8+ T cell effector mechanisms in resistance to infection. Annu. Rev. Immunol. 18:275-308.

3. Alcami, A., and Koszinowski, U.H. 2000. Viral mechanisms of immune evasion. Trends Microbiol. 8:410-418. 
4. Tortorella, D., Gewurz, B.E., Furman, M.H., Schust, D.J., and Ploegh, H.L. 2000. Viral subversion of the immune system. Annu. Rev. Immunol. 18:861-926.

5. Dick, T.P., Bangia, N., Peaper, D.R., and Cresswell, P. 2002. Disulfide bond isomerization and the assembly of MHC class I-peptide complexes. Immunity. 16:87-98.

6. Pamer, E., and Cresswell, P. 1998. Mechanisms of MHC class I-restricted antigen processing. Annu. Rev. Immunol. 16:323-358.

7. Goodfellow, P.N., et al. 1975. The beta2-microglobulin gene is on chromosome 15 and not in the HL-A region. Nature. 254:267-269.

8. Wiertz, E.J., et al. 1996. The human cytomegalovirus US11 gene product dislocates MHC class I heavy chains from the endoplasmic reticulum to the cytosol. Cell. 84:769-779.

9. Wiertz, E.J., et al. 1996. Sec61-mediated transfer of a membrane protein from the endoplasmic reticulum to the proteasome for destruction. Nature. 384:432-438

10. Ahn, K., et al. 1996. Human cytomegalovirus inhibits antigen presentation by a sequential multistep process. Proc. Natl. Acad. Sci. USA. 93:10990-10995.

11. Gruhler, A., Peterson, P.A., and Fruh, K. 2000. Human cytomegalovirus immediate early glycoprotein US3 retains MHC class I molecules by transient association. Traffic. 1:318-325.

12. Jones, T.R., et al. 1996. Human cytomegalovirus US3 impairs transport and maturation of major histocompatibility complex class I heavy chains. Proc. Natl. Acad. Sci. USA. 93:11327-11333.

13. Hill, A., et al. 1995. Herpes simplex virus turns off the TAP to evade host immunity. Nature. 375:411-415.

14. York, I.A., et al. 1994. A cytosolic herpes simplex virus protein inhibits antigen presentation to CD8+ T lymphocytes. Cell. 77:525-535.

15. Hengel, H., et al. 1997. A viral ER-resident glycoprotein inactivates the MHC-encoded peptide transporter. Immunity. 6:623-632.

16. Ahn, K., et al. 1997. The ER-luminal domain of the HCMV glycoprotein US6 inhibits peptide translocation by TAP. Immunity. 6:613-621.

17. Levitskaya, J., Sharipo, A., Leonchiks, A., Ciechanover, A., and Masucci, M.G. 1997. Inhibition of ubiquitin/proteasome-dependent protein degradation by the Gly-Ala repeat domain of the Epstein-Barr virus nuclear antigen 1. Proc. Natl. Acad. Sci. USA. 94:12616-12621.

18. Schwartz, O., Marechal, V., Le Gall, S., Lemonnier, F., and Heard, J.M. 1996. Endocytosis of major histocompatibility complex class I molecules is induced by the HIV-1 Nef protein. Nat. Med. 2:338-342.

19. Mangasarian, A., et al. 1997. The HIV-1 Nef protein acts as a connector with sorting pathways in the Golgi and at the plasma membrane. Immunity. 6:67-77.

20. Le Gall, S., et al. 1998. Nef interacts with the mu subunit of clathrin adaptor complexes and reveals a cryptic sorting signal in MHC I molecules. Immunity. 8:483-495.

21. Piguet, V., et al. 2000. HIV-1 Nef protein binds to the cellular protein PACS-1 to downregulate class I major histocompatibility complexes. Nat. Cell Biol. 2:163-167.

22. Stumptner-Cuvelette, P., et al. 2001. HIV-1 Nef impairs MHC class II antigen presentation and surface expression. Proc. Natl. Acad. Sci. USA. 98: $12144-12149$

23. Johnson, J.M., et al. 2001. Free major histocompatibility complex class I heavy chain is preferentially targeted for degradation by human T-cell leukemia/lymphotropic virus type 1 p12(I) protein. J. Virol. 75:6086-6094.

24. Trovato, R., et al. 1999. A lysine-to-arginine change found in natural alleles of the human T-cell lymphotropic/leukemia virus type 1 p12(I) protein greatly influences its stability. J. Virol. 73:6460-6467.

25. Joazeiro, C.A., and Weissman, A.M. 2000. RING finger proteins: mediators of ubiquitin ligase activity. Cell. 102:549-552.

26. Coscoy, L., and Ganem, D. 2000. Kaposi's sarcoma-associated herpesvirus encodes two proteins that block cell surface display of MHC class I chains by enhancing their endocytosis. Proc. Natl. Acad. Sci. USA. 97:8051-8056.

27. Coscoy, L., and Ganem, D. 2001. A viral protein that selectively downregulates ICAM-1 and B7-2 and modulates T cell costimulation. J. Clin. Invest. 107:1599-1606.

28. Ishido, S., Wang, C., Lee, B.S., Cohen, G.B., and Jung, J.U. 2000. Downregulation of major histocompatibility complex class I molecules by
Kaposi's sarcoma-associated herpesvirus K3 and K5 proteins. J. Virol. 74:5300-5309.

29. Haque, M., et al. 2001. Major histocompatibility complex class I molecules are down-regulated at the cell surface by the $\mathrm{K} 5$ protein encoded by Kaposi's sarcoma-associated herpesvirus/human herpesvirus-8. J. Gen. Virol. 82:1175-1180.

30. Coscoy, L., Sanchez, D.J., and Ganem, D. 2001. A novel class of herpesvirusencoded membrane-bound E3 ubiquitin ligases regulates endocytosis of proteins involved in immune recognition. J. Cell Biol. 155:1265-1273.

31. Lorenzo, M.E., Jung, J.U., and Ploegh, H.L. 2002. Kaposi's sarcoma-associated herpesvirus $\mathrm{K} 3$ utilizes the ubiquitin-proteasome system in routing MHC class I complexes to late endocytic compartments. J. Virol. 76:5522-5531.

32. Borodovsky, A., et al. 2001. A novel active site-directed probe specific for deubiquitylating enzymes reveals proteasome association of USP14. EMBOJ. 20:5187-5196.

33. van Kerkhof, P., Govers, R., Alves dos Santos, C.M., and Strous, G.J. 2000. Endocytosis and degradation of the growth hormone receptor are proteasome-dependent. J. Biol. Chem. 275:1575-1580.

34. Boname, J.M., and Stevenson, P.G. 2001. MHC class I ubiquitination by a viral PHD/LAP finger protein. Immunity. 15:627-636.

35. Dupre, S., Volland, C., and Haguenauer-Tsapis, R. 2001. Membrane transport: ubiquitylation in endosomal sorting. Curr. Biol. 11:R932-R934.

36. Thien, C.B., and Langdon, W.Y. 2001. Cbl: many adaptations to regulate protein tyrosine kinases. Nat. Rev. Mol. Cell Biol. 2:294-307.

37. Pickart, C.M. 2001. Ubiquitin enters the new millennium. Mol. Cell. 8:499-504.

38. Guerin, J.L., et al. 2002. Myxoma virus leukemia-associated protein is responsible for major histocompatibility complex class I and Fas-CD95 down-regulation and defines scrapins, a new group of surface cellular receptor abductor proteins. J. Virol. 76:2912-2923.

39. Bonifacino, J.S. 1996. Reversal of fortune for nascent proteins. Nature. 384:405-406.

40. Brodsky, J.L., and McCracken, A.A. 1999. ER protein quality control and proteasome-mediated protein degradation. Semin. Cell Dev. Biol. 10:507-513.

41. Plemper, R.K., and Wolf, D.H. 1999. Retrograde protein translocation: ERADication of secretory proteins in health and disease. Trends Biochem. Sci. 24:266-270.

42. Kikkert, M., et al. 2001. Ubiquitination is essential for human cytomegalovirus US11-mediated dislocation of MHC class I molecules from the endoplasmic reticulum to the cytosol. Biochem. J. 358:369-377.

43. Shamu, C.E., Flierman, D., Ploegh, H.L., Rapoport, T.A., and Chau, V. 2001. Polyubiquitination is required for US11-dependent movement of $\mathrm{MHC}$ class I heavy chain from endoplasmic reticulum into cytosol. $\mathrm{Mol}$. Biol. Cell. 12:2546-2555.

44. Shamu, C.E., Story, C.M., Rapoport, T.A., and Ploegh, H.L. 1999. The pathway of US11-dependent degradation of MHC class I heavy chains involves a ubiquitin-conjugated intermediate. J. Cell Biol. 147:45-58.

45. Ye, Y., Meyer, H.H., and Rapoport, T.A. 2001. The AAA ATPase $\mathrm{Cdc} 48 / \mathrm{p} 97$ and its partners transport proteins from the ER into the cytosol. Nature. 414:652-656.

46. Furman, M.H., Ploegh, H.L., and Tortorella, D. 2002. Membrane-specific, host-derived factors are required for US2- and US11-mediated degradation of major histocompatibility complex class I molecules. J. Biol. Chem. 277:3258-3267.

47. Story, C.M., Furman, M.H., and Ploegh, H.L. 1999. The cytosolic tail of class I MHC heavy chain is required for its dislocation by the human cytomegalovirus US2 and US11 gene products. Proc. Natl. Acad. Sci. USA. 96:8516-8521.

48. Gewurz, B.E., Gaudet, R., Tortorella, D., Wang, E.W., and Ploegh, H.L. 2001. Virus subversion of immunity: a structural perspective. Curr. Opin. Immunol. 13:442-450.

49. Gewurz, B.E., et al. 2001. Antigen presentation subverted: structure of the human cytomegalovirus protein US2 bound to the class I molecule HLA-A2. Proc. Natl. Acad. Sci. USA. 98:6794-6799.

50. Fiebiger, E., Story, C., Ploegh, H.L., and Tortorella, D. 2002. Visualization of the ER-to-cytosol dislocation reaction of a type I membrane protein. EMBO J. 21:1041-1053. 